\title{
BRAZILIAN OUTLOOK OF BREAST CANCER MORTALITY IN VALIDITY OF MAMMOGRAPHIC SCREENING - A TIME REVIEW
}

Letícia Passos Souza', Sabrina Santos Alves, Ícaro García Viana

'Universidade Federal da Bahia, Instituto Multidisciplinar em Saúde, Campus Anísio Teixeira - Vitória da Conquista (BA), Brazil.

Introduction: Breast Cancer is the cancer with the highest incidence among the female population at national and worldwide levels, except for non-melanoma skin cancer. It is also the main cause of cancer deaths in women, representing an important public health problem. This scenario justifies the implementation of strategies that aim to reduce mortality and morbidity rates in its target population. Based on this demand, the document "Breast Cancer Control - Consensus Document" was prepared in 2004 by the Brazilian National Cancer Institute (INCA) and the Brazilian Department of Health, with an improvement in 2015 through the "Guidelines for Early Detection of Breast Cancer in Brazil". Objectives: The objective of the present work is to evaluate the outlook of breast cancer mortality in women in Brazil between the years 2009 and 2018, considering the mammographic screening implementation. Methods: A retrospective and descriptive epidemiological study was carried out using the last ten years of Brazilian data available on INCA's Online Mortality Atlas (from 2009 to 2018). In addition, documents released by INCA and the Department of Health that contemplate breast cancer issues in women and mammographic screening were selected, as they reflect the reality of the country. The age group primarily observed was between 50 and 69 years, which correspond to that indicated for mammographic screening in the current legislation. Results: Deaths due to breast cancer rose from 11,968 to 17,572 between 2009 and 2018, which represents an increase of $46.82 \%$, as shown in Table 1. Considering the same time frame, the values in percentage show an increase of $0.48 \%$ when compared to the total number of deaths (from $2.54 \%$ to $3.02 \%$ - as shown in Table 2). The mortality rate by age group increased: from 32.54 to 35.63 for the group aged 50 to 59 and from 41.78 to 53.63 for the group aged 60 to 69 years. The data show the persistent increase in the mortality rate due to the disease, despite the implementation of mammographic screening. The significant reduction in mortality predicted in the literature for the age group 50 to 69 years depends on factors such as high coverage and quality of screening, in addition to adequate treatment. Globalization and industrialization increased exposure to risk factors. The increase in life expectancy is expected to interfere with incidence and prevalence. Conclusions: Conditions mentioned above may justify the increase in mortality. Therefore, the relevance of screening in cancer's pre-clinical identification still justifies its indication and implantation. 